\title{
Vaccine-elicited murine antibody WS6 neutralizes diverse beta-coronaviruses by recognizing a helical stem supersite of vulnerability
}

Wei Shi ${ }^{1,3}$, Lingshu Wang ${ }^{1,3}$, Tongqing Zhou ${ }^{1,3}$, Mallika Sastry ${ }^{1}$, Eun Sung Yang ${ }^{1}$, Yi Zhang ${ }^{1}$, Man Chen ${ }^{1}$, Xuejun Chen ${ }^{1}$, Misook Choe ${ }^{1}$, Adrian Creanga ${ }^{1}$, Kwan Leung ${ }^{1}$, Adam S. Olia ${ }^{1}$, Amarendra Pegu $^{1}$, Reda Rawi ${ }^{1}$, Chen-Hsiang Shen ${ }^{1}$, Erik-Stephane D. Stancofski ${ }^{1}$, Chloe Adrienna Talana ${ }^{1}$, I-

${ }^{1}$ Vaccine Research Center, National Institute of Allergy and Infectious Diseases, National 17 Institutes of Health, Bethesda, MD 20892, USA

$18{ }^{2}$ Electron Microscopy Laboratory, Cancer Research Technology Program, Leidos Biomedical

19 Research, Inc., Frederick National Laboratory for Cancer Research, Frederick, MD 21702, USA.

$20 \quad{ }^{3}$ These authors contributed equally

$21{ }^{4}$ Lead Contact

* Correspondence: jmascola@nih.gov (J.R.M.), pdkwong@nih.gov (P.D.K.)

Keywords: beta-coronavirus, broadly neutralizing antibody, COVID-19, crystal structure, SARS- 


\section{Summary}

34 Immunization with SARS-CoV-2 spike elicits diverse antibodies, but can any of these neutralize

35 broadly? Here, we report the isolation and characterization of antibody WS6, from a mouse

36 immunized with mRNA encoding the SARS-CoV-2 spike. WS6 bound diverse beta-coronavirus

37 spikes and neutralized SARS-CoV-2 variants, SARS-CoV, and related sarbecoviruses. Epitope

38 mapping revealed WS6 to target a region in the S2 subunit, which was conserved among SARS-

39 CoV-2, MERS-CoV, and hCoV-OC43. The crystal structure at 2- $\AA$ resolution of WS6 with its S2

40 epitope revealed recognition to center on a conserved helix, which was occluded in both prefusion

41 and post-fusion spike conformations. Structural and neutralization analyses indicated WS6 to

42 neutralize by inhibiting fusion, post-viral attachment. Comparison of WS6 to other antibodies

43 recently identified from convalescent donors or mice immunized with diverse spikes indicated a

44 stem-helical supersite - centered on hydrophobic residues Phe1148, Leu1152, Tyr1155, and

45 Phe1156 - to be a promising target for vaccine design. 


\section{$48 \quad$ Highlights}

- SARS-CoV-2 spike mRNA-immunized mouse elicited an antibody, WS6, that cross reacts with spikes of diverse human and bat beta-coronaviruses

- WS6 neutralizes SARS-CoV-2 variants, SARS-CoV, and related viruses

- Crystal structure at 2- $\AA$ resolution of WS6 in complex with a conserved S2 peptide reveals recognition of a helical epitope

- WS6 neutralizes by inhibition of fusion, post-viral attachment

- WS6 recognizes a supersite of vulnerability also recognized by other recently identified antibodies

- Helical supersite of vulnerability comprises a hydrophobic cluster spanning three helical turns, with acid residues framing the center turn

- Genetic and structural analysis indicate supersite recognition to be compatible with diverse antibody ontogenies 


\section{Introduction}

64

The COVID-19 pandemic, resulting from the zoonotic infection of severe acute respiratory syndrome coronavirus 2 (SARS-CoV-2), continues to rage on, fueled by continuously evolving variants, which are making current licensed vaccines less effective (Araf et al., 2022; Doria-Rose et al., 2021; Edara et al., 2021; Garcia-Beltran et al., 2022; Liu et al., 2021). Vaccines capable of neutralizing all SARS-CoV-2 variants for the foreseeable future are of high interest. Antibodies with broad neutralizing capacity are also of interest: if ultrapotent, they might be useful as therapeutic antibodies; but even if only moderate potency, their epitopes are useful as vaccine templates (Kong et al., 2016).

Virtually all neutralizing antibodies are directed against the trimeric ectodomain of the spike glycoprotein, which comprises two subunits S1 and S2. Neutralizing antibodies isolated from COVID-19 convalescent donors or from vaccinees after spike immunization are directed primarily against the N-terminal domain (NTD) or receptor-binding domain (RBD) on the S1 subunit of the trimeric viral surface spike glycoprotein (Spike) (Barnes et al., 2020; Brouwer et al., 2020; Cao et al., 2020; Cerutti et al., 2021; Ju et al., 2020; Liu et al., 2020; McCallum et al., 2021; Robbiani et al., 2020; Rogers et al., 2020; Seydoux et al., 2020; Suryadevara et al., 2021; Zost et al., 2020). Evolving SARS-CoV-2 variants, such as Delta and Omicron, evade these antibodies by mutations that reduce or knockout antibody binding, but maintain or even enhance infectivity (Garcia-Beltran et al., 2022; Liu et al., 2021; Sievers et al., 2022; Syed et al., 2022). Antibodies against most other regions on the spike are generally poorly neutralizing to non-neutralizing; several antibodies, however, such as antibody S2P6 (Pinto et al., 2021) have been reported to neutralize diverse strains of beta coronaviruses through recognition of a stem-helix supersite of vulnerability in the S2 subunit (Hsieh et al., 2021; Li et al., 2022; Sauer et al., 2021; Zhou et al., 2021). 
To investigate the breadth of neutralizing antibodies obtained from mice vaccinated by mRNA encoding the SARS-CoV-2 spike, we assessed monoclonal antibodies for the location of their epitopes, the breadth of their binding to diverse spikes, and their neutralization capacities. We found one, antibody WS6, with broad binding capacity and moderate neutralization potency, and we determined its crystal structure in complex with its epitope, the step in the entry pathway where it neutralized, and how its recognition compared with other recently identified antibodies with overlapping epitopes. The results reveal a highly promising vaccine target in the S2 subunit comprising a hydrophobic cluster spanning three helical turns, with acidic residues framing its center turn - and add WS6 to the panel of antibodies by which to guide its vaccine development.

\section{Results}

\section{Identification and characterization of SARS-CoV-2 spike-specific antibodies from}

To obtain antibodies specific for SARS-CoV-2 spike glycoprotein, we immunized mice with mRNA coding for SARS-CoV-2 spike (Figure 1A). To generate hybridomas, we boosted with soluble spike protein and after three days generated hybridomas by fusing splenocyte B cells with Sp2/0 cells from the mouse with the highest plasma neutralization titers to SARS-CoV-2. Eleven monoclonal antibodies, named WS1 to WS11, bound SARS-CoV-2 S-dTM by ELISA

(Figure 1B). Nine of these bound the S1 subunit, either S1-short1 (spike residues 1-670) or S1R (residues 1-537). Six of them, WS1, WS2, WS3, WS7, WS8, and WS10, bound NTD; and three of them, WS4, WS9, and WS11, bound RBD. Antibodies WS5 and WS6, however, did not bind NTD, RBD, or S1, and their binding epitopes were presumably on the S2 subunit of the spike. 
To provide insight into the breadth of binding, we assessed recognition of WS1-11 on a

109 panel of prefusion-stabilized diverse beta-coronavirus spikes (S2Ps) (Wrapp et al., 2020).

110 Detectable binding was observed against SARS-CoV-2, SARS-CoV, MERS-CoV and hCoV-

111 HKU1 for five antibodies (Figure 1C). For antibodies WS3, WS4, and WS7, binding was more

112 than 1,000-times weaker against the divergent strain versus that of the immunogen. However, for

113 antibodies WS5 and WS6, binding was only about 10-fold reduced versus SARS-CoV-2.

114 Neutralization assessments revealed that WS5 neutralized neither SARS-CoV-2 nor SARS-CoV,

115 whereas WS6 could neutralize both (Figure 1D). WS6 was further studied for its epitope and

116 neutralization activities.

\section{WS6 recognizes diverse beta-coronaviruses spikes and neutralizes SARS-CoV-2 and} variants, SARS-CoV, and related viruses from bat, pangolin, and other animals

To assess fully the broad reactivity of WS6, we performed ELISAs against S2Ps from an even more divergent panel of coronaviruses, including RaTG13, WIV1, SHC014, hCoV-OC43,

122 and hCoV-229E (Figure 2A). Remarkably, WS6 was able to bind all beta-coronaviruses tested,

123 though not to the alpha-coronavirus hCoV-229E (Figure S1). The apparent binding affinities of

124 WS6 to S2Ps, measured by biolayer interferometry (BLI), showed nanomolar or lower dissociation

125 constants $\left(\mathrm{K}_{\mathrm{D}} \mathrm{s}\right)$, with slow dissociation such that $\mathrm{k}_{\mathrm{off}}$ values could not be determined accurately in

126 many cases (Figure 2B). To determine if WS6 could bind spike proteins on cell surface, we

127 expressed spikes of SARS-CoV-2, SARS-CoV, MERS-CoV, hCoV-HKU1, and hCoV-OC43 on

128 the surface of Expi-293 cells and analyzed WS6 binding by flow cytometry. We found that WS6

129 bound well to the cell surface expressing the beta-coronavirus spikes (Figures 2C and S2). 
To determine if the broad recognition of WS6 for spikes translated into broad neutralization of beta-coronaviruses, we assessed its neutralization activities by pseudovirus neutralization assays (Naldini et al., 1996; Wang et al., 2021). WS6 neutralized all tested variants of SARS-CoV-2 including Omicron (B.1.1.529), with $\mathrm{IC}_{50} 2.46-26.52 \mu \mathrm{g} / \mathrm{ml}$ (Figures 2D and S3; Table S1). WS6 could also neutralize beta-coronaviruses related to SARS-CoV-2 (such as RaTG13, Pangolin_GD, and Pangolin_GX), SARS-CoV, and related viruses Frankfurt1, Civet007-2004, WIV1, and CoV, consistent with its lower ELISA binding to this strain (Table S1).

SHC014 with $\mathrm{IC}_{50} 0.11-4.91 \mu \mathrm{g} / \mathrm{ml}$ (Figures 2D and S3; Table S1). WS6 also neutralized related beta-coronaviruses, such as RaTG13, Pangolin-GX, Civet007-2004, and SHC014, with sub micromolar $\mathrm{IC}_{50}$, better than against SARS-CoV-2 despite being elicited by immunizations with SARS-CoV-2 spike. The only tested beta-coronavirus strain WS6 failed to neutralize was MERS-

\section{WS6 epitope mapping}

We attempted to map the epitope of WS6 by visualizing its recognition of the spike ectodomain by negative strain-electron microscopy (EM). 2D-classification of antigen-binding fragment (Fab) of WS6 in complex with the S2P spike (WA-1 strain) showed generally unbound 146 spikes, with only $4 \%$ of the images yielding a trimer with Fabs binding in the membrane-proximal S2 stem (Figure 3A). We also analyzed Fab WS6 in complex with spike S2 subunit; 2Dclassification indicated WS6 to bind S2, though most of S2 appeared to be disordered (Figure 3B). To further map the epitope, we performed peptide array-based epitope mapping and identified a 17-residue peptide, PELDSFKEELDKYFKNH (SARS-CoV-2 spike residues 1143-

151 1159) to bind WS6 (Figure 3C), suggesting WS6 epitope to be at the S2 stem-helix region of the 152 spike near the viral membrane, consistent with our negative stain-EM observation. This peptide is 
conserved among SARS-CoV, SARS-CoV-2, and RaTG13, and is mostly conserved among diverse beta-coronaviruses (Figure S4A).

\section{Crystal structure of WS6 in complex with a conserved S2 peptide}

To elucidate the mechanism for the broad reactivity of WS6, we determined a crystal structure at $2 \AA$ of Fab WS6 in complex with peptide Ace-F ${ }_{1148}$ KEELDKYFK $_{1157}$-PEG12-LysBiotin (residue numbers were based on the SARS-CoV-2 spike sequence) (Figure 3D and Table

S2). The peptide contained a 10-residue segment conserved among beta-coronaviruses (Figure S4A) and was acetylated at $\mathrm{N}$ terminus and biotin-pegylated at $\mathrm{C}$ terminus. Well-defined electron density was observed for the entire WS6 Fab and for the entire peptide, including the acetyl group at the N terminus and part of the polyethylene glycol at the C terminus (Figure 3D). Peptide binding interactions involved both heavy and light chains, with heavy chain contributing $\sim 360 \AA^{2}$ buried surface area (BSA) and light chain contributing $\sim 230 \AA^{2}$ (Table S3). The BSA of the peptide was slightly larger at $\sim 675 \AA^{2}$, not counting the BSA of the visible PEG fragment $\left(\sim 100 \AA^{2}\right.$ total). This binding interface is smaller than a typical antibody-binding epitope, indicating the full WS6 epitope to likely involve additional residues.

All complementarity-determining regions (CDRs) of both heavy and light chains were involved in binding, creating a groove that cradled the epitope (Figure 3D). In the WS6-bound crystal structure, the S2 epitope formed a three-turn $\alpha$ helix; examination of its binding mode revealed binding for a longer helix with extensions on both termini without any major clashes. Most 173 of the residues of the peptide involved in binding, except for Glu1150 and Lys1154, which were on 174 the side of the helix facing WS6 (Figure 3E). Aromatic or hydrophobic residues, Phe1148, 
interactions with $\operatorname{Arg}_{50}$ and $\operatorname{Tyr} 91_{\mathrm{L}}$ of WS6 light chain and hydrophobic interactions with Ser97 $\mathrm{H}$ of heavy chain. Tyr1155 side chain stuck into a cavity form by CDR L3 from Tyr91 ${ }_{L}$ to Pro96 $6_{L}$ and had aromatic or hydrophobic interactions with side chains of $\operatorname{Tyr} 91_{\mathrm{L}}, \operatorname{Tyr} 94_{\mathrm{L}}$, and $\operatorname{Pro}_{\mathrm{L}} \mathrm{L}$ and the peptide backbone. Leu1152 and Phe1156 side chains bound in a pocket between heavy and light

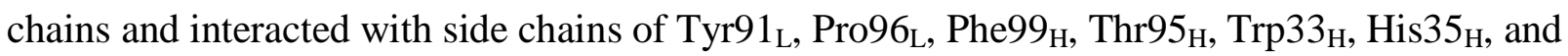
$\mathrm{Tyr}_{4} 7_{\mathrm{H}}$ (Figure 3E and 3F and Table S3).

\section{WS6 neutralizes by inhibition of fusion steps post viral attachment}

To elucidate the mechanism by which WS6 neutralize beta-coronaviruses, we incubated BHK21-ACE2 cells with SARS-CoV-2 spike pseudotyped lentivirus on ice to allow virus to attach to ACE2. After thorough washing, cells were incubated with WS6 or WS4 on ice for one hour and at

$37 \square$ for 72 hours. WS4, an RBD-directed neutralizing antibody, could neutralize not more than $40 \%$ of the virus, whereas WS6 had no problem neutralizing the ACE2 pre-attached virus (Figure 4A). We performed a similar experiment using 293 flpin-TMPRSS2-ACE2 cells for WS6 and S2P6 (Pinto et al., 2021), which also binds to the S2-stem helix, and found that both antibodies could neutralize ACE2 pre-attached virus. These results suggest that WS6 is likely to neutralize SARSCoV-2 by engaging in steps post-viral attachment to ACE2.

As described above in the structural analysis, WS6 recognized the hydrophobic face of the stem helix with residues Phe1148, Leu1152, Tyr1155, and Phe1156 binding in the center of the paratope groove. In the prefusion structure of SARS-CoV-2 spike (PDB: 6xr8) (Cai et al., 2020; Ke et al., 2020), these hydrophobic side chains pack in the coiled-coil interface of the 3-helical bundle. Modeling of WS6 binding to the prefusion SARS-CoV-2 spike, based on the WS6-peptide complex, revealed substantial clashes (Figure 4B, left panel), indicating that WS6 binding would require 
unpacking or disassembly of the helical bundle. Similarly, the postfusion spike structure (PDB: 6xra) (Cai et al., 2020) was incompatible with binding of WS6 to the hydrophobic side of the peptide helix as revealed by the crystal structure, because these hydrophobic residues are involved in the packing of the small helix with the central coiled coil in the postfusion structure (Figure 4B, right panel). Because the spike binding to the receptor and transitioning from the prefusion conformation to postfusion conformation drive the membrane fusion process, WS6 maybe even more apt to bind during this conformational transition and inhibit the fusion process (Figure 4C).

\section{A helical stem supersite of vulnerability}

Several broad beta-coronavirus-neutralizing antibodies have been identified recently that target the S2 stem-helix region, including the afore mentioned S2P6 (Pinto et al., 2021) as well as antibodies B6 (Sauer et al., 2021), IgG22 (Hsieh et al., 2021), CCP40.8 (Zhou et al., 2021), and CV3-25 (Li et al., 2022). Superposition of their S2 helical epitopes indicated these antibodies to bind to the stem helix with varying orientations (Figure 5A) and to target different sets of residues ranging from residue position 1142 to 1164 on S2 (Figures 5B and S5). Epitope analysis indicated a common subset of residues, namely Phe1148, Lys1149, Glu1153, Leu1152, Asp1153, Tyr1155, and Phe1156, to interact with four of the six antibodies (Figure 5B, left). These residues, which spanned three helical turns on S2 with a central hydrophobic cluster sandwiched by hydrophilic residues Lys1149, Glu1151, and Asp1153, formed a supersite of vulnerability for antibody

218 recognition (Figure 5B, middle and right). Sequence analysis indicated this S2 supersite to be

219 highly conserved among beta-coronaviruses (Figure 5C), providing the basis for the broad

220 neutralization by WS6 and other antibodies targeting this site. Overall, these S2 stem-helix

221 antibodies are likely to share a similar mechanism of neutralization. Before or even after spike 
binds to ACE2, the stem helix appears to adopt a conformation or conformations that expose this supersite, allowing for the stem-helix antibodies to bind and prevent spike conformations needed for fusion, thereby stalling the entry process. Importantly, these S2-helix directed broad neutralizers have diverse origin genes, except for B6 and $\operatorname{IgG} 22$, which utilize the same VH gene and appears to be of the same antibody class (Figure S4B). In addition, the antibodies have only low-to-moderate somatic hypermutation, suggesting diverse ontogenies are possible with little barrier to their development (Figure S4).

\section{Discussion}

Zoonotic infections from beta-coronaviruses have caused multiple pandemics and endemics in recent years, including SARS (Drosten et al., 2003; Ksiazek et al., 2003), Middle East respiratory syndrome (MERS) (Zaki et al., 2012), and the ongoing COVID-19 (Zhou et al., 2020a; Zhu et al., 2020). It seems likely that additional beta-coronavirus zoonotic pandemics will occur in the future. Broadly neutralizing antibodies or broad vaccines against a wide spectrum of betacoronaviruses may be effective at preventing or ameliorating such pandemics - and could even be of use against the current COVID-19, if variants do succeed in escape control by current vaccines. In this study, we isolated an S2-directed antibody, named WS6, from a mouse immunized with mRNA-encoded SARS-CoV-2 spike. WS6 could bind and neutralize all variants of SARS-CoV-2, including Delta and Omicron; it could also neutralize SARS-CoV, as well as bat, civet, and pangolin beta-coronaviruses related to SARS-CoV and SARS-CoV-2. Crystal structure of WS6 in complex with S2 stem-helix epitope peptide revealed the structural basis for broad recognition and, together with neutralization and binding analyses, suggest a potential mechanism by which WS6 neutralize broadly beta-coronaviruses. 
Several other groups have recently reported S2-directed antibodies including antibodies S2P6 (Pinto et al., 2021), CC40.8 (Zhou et al., 2021) and CV3-25 (Li et al., 2022) from SARSCoV-2-infected convalescent donors and B6 (Sauer et al., 2021) and IgG22 (Hsieh et al., 2021) from vaccinated mice. We measured directly the neutralization of both WS6 and S2P6 to diverse SARS-CoV-2 variants and to diverse coronaviruses generally (Figure S3). In light of its high breadth, we were surprised to find S2P6 was unable to neutralize the Omicron variant of SARSCoV-2, although WS6 did neutralize Omicron. WS6 did, however, neutralize all tested strains more potently than S2P6, except for MERS-CoV, against which WS6 was non-neutralizing. In terms of the murine antibodies, the recognition of antibodies B6 and IgG22 was very similar, both utilizing the same heavy chain origin gene (VH1-19) and both generated after immunization with spikes from SARS-CoV-2 and MERS-CoV (Hsieh et al., 2021; Pinto et al., 2021). By contrast, WS6 did not use the same V-gene and was generated only by immunization with the SARS-CoV-2 spike - thereby revealing a new mode of murine S2-helix recognition and showing MERS-CoV spike immunization was no required to elicit these antibodies.

It will be interesting to determine whether S2-helix peptide-based immunizations can focus the immune response, enabling the elicitation of broadly neutralizing serological responses, as has been done with HIV-1 fusion peptide (Kong et al., 2019; Xu et al., 2018). Nanoparticles displaying the S2-helix may also be helpful in focusing the immune response, as has been done with the influenza stem cite of vulnerability (Boyoglu-Barnum et al., 2021; Kanekiyo et al., 2013; Yassine et al., 2015). Whether S2-helix focused immunization will enable sufficiently broad and potent responses to enable protection from future SARS-CoV-2 variants or to future beta-coronavirus

266 zoonotic crossovers remains to be seen. It seems likely that such immune focusing can be carried 
267 out in conjunction with spike-based immunizations, perhaps using currently licensed vaccines

268 from Moderna, Pfizer and Johnson and Johnson, all of which incorporate the S2-helical region.

270 Acknowledgements

271 We thank R. Andrabi for providing CC40.8 coordinates, B. Graham for suggesting use of flpin-

272 TMPRSS2-ACE2 cells, T. Stephens for assistance with negative-stain EM, J. Stuckey for

273 assistance with figures, and members of the Virology Laboratory, Vaccine Research Center, for

274 discussions and comments on the manuscript. Support for this work was provided by the

275 Intramural Research Program of the Vaccine Research Center, National Institute of Allergy and

276 Infectious Diseases, National Institutes of Health and by federal funds from the Frederick National

277 Laboratory for Cancer Research under Contract HHSN261200800001E. Use of sector 22

278 (Southeast Region Collaborative Access team) at the Advanced Photon Source was supported by

279 the US Department of Energy, Basic Energy Sciences, Office of Science, under contract number

280 W-31-109-Eng-38.

\section{Author Contributions}

283 W.S. isolated antibodies WS1-WS11 and performed ELISA binding assays; L.W. headed

284 neutralization studies, prepared spike plasmids and performed cell surface binding assays; T.Z.

285 determined and analyzed the crystal structure of WS6-peptide complex, and wrote manuscript;

286 M.S. performed Octet binding assays, provided spike proteins for ELISA, and assisted with

287 obtaining peptide for crystallization; E.S.Y., Y.Z., and M.Chen performed WS6 neutralization of

288 SARS related viruses; X.C. provided mouse NGS sequence data; M.Choe provided S2P6 antibody;

289 A.C. provided 293 flpin-TMPRSS2-ACE2 cells; K.L. assisted with preparation of pseudoviruses; 
290 A.S.O provided SARS-CoV-2 S2P-His for Octet measurements; A.P. and C.A.T performed cell

291 surface staining; R.R. assisted with obtaining Peptide for crystallization; C.-H.S. prepared

292 phylogenetic tree; E.-S.S. crystallized the protein complex; I.-T.T. performed transfection for Beta-

293 CoV and S2 antibody productions; S.W. wrote manuscript and performed PISA analysis of

294 antibody-peptide interface; K.S.C carried out mouse immunization experiment; Y.T. performed

295 negative-stain EM analysis; J.R.M. supervised antibody isolation, neutralization assessments, and

296 cell surface binding assays; P.D.K. oversaw the project and - with S.W and T.Z - wrote the

297 manuscript, with all authors providing comments or revisions.

\section{Competing interest declaration}

300 The authors declare no competing interest. 


\section{METHODS}

304 RESOURCE AVAILABILITY

305 Materials Availability

306 Plasmids generated in this study are available upon request.

\section{Data and Code Availability}

Crystal diffraction data and structure coordinates of WS6 in complex have been deposited with the

Protein Data Bank, PDB: 7TCQ, DOI: 10.2210/pdb7tcq/pdb.

\section{EXPERIMENTAL MODEL AND SUBJECT DETAILS}

\section{3}

\section{Mouse Studies}

Animal experiments were carried out in compliance with all pertinent US National Institutes of Health regulations and approval from the Animal Care and Use Committee (ACUC) of the Vaccine Research Center. BALB/cJ mice (Jackson Laboratory), 6- to 8-week-old female, were used.

\section{Cell Lines}

FreeStyle 293-F (cat\# R79007) and Expi293F cells (cat\# A14528; RRID: CVCL_D615) were purchased from ThermoFisher Scientific Inc. FreeStyle 293-F cells were maintained in FreeStyle 293 Expression Medium, while Expi293F cells were maintained in Expi Expression Medium. The above cell lines were used directly from the commercial sources and cultured according to manufacturer suggestions. 


\section{METHOD DETAILS}

327

328

329

330

331

332

333

334

335

336

337

338

339

\section{Mouse immunization, hybridoma generation and $\mathrm{mAb}$ isolation}

Mice were immunized with mRNA coding for SARS-CoV-2 spike of the wildtype WA-1 strain at weeks 0 and 3 intramuscularly, utilizing methods described previously (Corbett et al., 2020). One mouse with the best neutralizing antibody titer against SARS-CoV-2 spike was boosted intravenously with $20 \square \mu \mathrm{g}$ of SARS-CoV-2 S-dTM at week 12 . Three days later, splenocytes were harvested and fused with Sp2/0 myeloma cells (ATCC) using polyethylene glycol (PEG) 1450 (50\% (w/v), Sigma-Aldrich) according to the standard methods. Cells were cultured and screened in RPMI complete medium that contained $20 \%$ FCS and $1 \times 100 \square \mu \mathrm{M}$ hypoxanthine, $0.4 \square \mu \mathrm{M}$ aminopterin and $16 \square \mu \mathrm{M}$ thymidine (Sigma-Aldrich). Supernatants from resulting hybridomas were screened for binding, using ELISA, to SARS-CoV-2- S1, NTD, RBD or S-dTM as well as for neutralizing activity. Subclones were generated by limiting dilution. After three rounds of screening and subcloning, stable antibody-producing clones were isolated and adapted to hybridoma-serum-free medium (Life Technologies Corp., Grand Island, NY, USA). Supernatants were collected from selected hybridoma clones and purified through a protein G-sepharose column (GE Healthcare). mAbs were isotyped with Pierce rapid isotyping kit (Cat\#26178). mAb Fabs were generated using Pierce Fab kit (Cat\#44985) following manufacturer's instructions. mAb and Fab purity was confirmed by SDS-PAGE. Selected hybridoma were sent to GenScript (Piscataway, NJ 08854, USA) for hybridoma heavy and light chain variable sequences and IMGT analysis.

\section{ELISA and dot-blot}


ELISA plates were coated with the SARS-CoV-2 proteins, S-dTM, S1-short (residues 1-670), S1R (residues 1-537), RBD, and NTD, at $1 \square \mu \mathrm{g} / \mathrm{ml}$ in PBS at $4 \square{ }^{\circ} \mathrm{C}$ overnight. After standard washes and blocks, plates were incubated with 50ul/each well, serial dilutions of mAbs for one hour at room temperature. Anti-mouse whole IgG horseradish peroxidase conjugates (Jackson Laboratory) were used as secondary antibodies, and 3,5,3'5'-tetramethylbenzidine (TMB) (KPL, Gaithersburg, MD) was used as the substrate.

To identify WS6 epitope, a dot-blot was performed. SARS-CoV spike peptide array (NR52418), was purchased from BEI resource. Seventy-eight peptides (\#91-169) from the peptide array which covers SARS-CoV spike S2 region, were dotted on a nitrocellulose membrane respectively, 20ng/2ul/each dot. SARS-CoV-2 RBD, S2, S-dTM and S2P proteins were also dotted on the membrane as controls. Block non-specific sites by soaking in 5\% dry milk in PBS-Tween 20. The membrane was washed three times with PBS-T buffer, then incubated with $2 \mathrm{ug} / \mathrm{ml}$ of mAb, WS6 in blocking buffer for one hour at room temperature. After wash three times with PBST buffer, the membrane was incubated half hour with anti-mouse IgG horseradish peroxidase conjugates (Jackson Laboratory) as secondary antibody. Then membrane was developed with ECL medium.

\section{Expression and purification of beta-coronavirus spike trimer proteins}

Diverse beta-coronavirus spike soluble proteins were stabilized in prefusion conformation by double-proline mutations corresponding to K986P and V987P in SARS CoV-2 spike protein, along with a T4-phage fibritin trimerization domain (foldon) at the $\mathrm{C}$ terminus (Wrapp et al., 2020) followed by an HRV3C cleavage site, His8 and Twin-Streptactin purification tags. Additionally, the furin cleavage sites between S1 and S2 were mutated; for HKU1 and OC43, RRKRR was 
replaced by GGSGG; for MERS-CoV, RSVR was replaced with ASVG; for SARS-CoV, SHC014, WIV1, and RaTG13, SLLRST was replaced with SLLAST. DNA sequences encoding diverse beta-coronavirus S2P proteins were cloned into mammalian expression vector $\mathrm{pVRC} 8400$ and the proteins expressed by transient transfection of FreeStyle 293-F cells as previously described (Zhou et al., 2020b). Specifically, $1 \mathrm{mg}$ of transfection grade plasmid DNA and $3 \mathrm{ml}$ of Turbo293 transfection reagent (Speed BioSystems) each in 20ml Opti-MEM (Thermo Fisher Scientific), were pre-mixed and transfected into FreeStyle 293-F cells. Transfected cells were grown for 6 days at $37^{\circ} \mathrm{C}$, and the supernatant was harvested by centrifugation to remove cell debris. Supernatants were sterile-filtered, and the spike trimers were purified by nickel affinity chromatography using cOmplete His-Tag purification resin. The resin was washed with $50 \mathrm{mM}$ Tris, $400 \mathrm{mM} \mathrm{NaCl}, 10 \mathrm{mM}$ and $25 \mathrm{mM}$ Imidazole $\mathrm{pH} 8.0$ buffer. The proteins were eluted in 50 $\mathrm{mM}$ Tris $\mathrm{pH}$ 8.0, $400 \mathrm{mM} \mathrm{NaCl}, 300 \mathrm{mM}$ Imidazole. Protein fractions were pooled and further purified by size-exclusion chromatography (SEC) using a Superdex 200 16/600 column (Cytiva) in PBS. Fractions corresponding to trimeric spike proteins were pooled, concentrated to $1 \mathrm{mg} / \mathrm{ml}$.

\section{Production of antibodies}

Antibody heavy and light variable region gene sequences were synthesized (Gene Universal Inc,

Newark DE) and subcloned into corresponding pVRC8400 vectors (https://www.addgene.org).

The resulting plasmids of heavy and light chain pairs were co-transfected in Expi293F cells

(Thermo Fisher) using Turbo293 transfection reagent (Speed BioSystems) as described previously

391 (Wu et al., 2011). On day 6 post transfection, the culture supernatants were harvested, sterile

392 filtered and loaded onto a protein A column. The columns were washed with PBS, and IgG

393 proteins were eluted with a low pH IgG elution buffer (Pierce) and immediately neutralized with 
$1 \mathrm{M}$ Tris- $\mathrm{HCl} \mathrm{pH}$ 8.0. Purified IgGs were subsequently dialyzed twice against PBS pH 7.4 using 10 $\mathrm{kD}$ dialysis cartridges (Pierce) and used for measurements.

\section{Full-length spike constructs}

Codon optimized cDNAs encoding full-length spike from SARS-CoV-2 (GenBank ID:

QHD43416.1) were synthesized, cloned into the mammalian expression vector VRC8400

(Barouch et al., 2005) and confirmed by sequencing. Spike containing D614G amino acid change was generated using the wildtype spike sequence. Other variants containing single or multiple amino-acid changes in the spike gene from wildtype or D614G were made by mutagenesis using

E484K-N501Y-D614G-H655Y-T1027I-V1176F), B.1.617.2 ( T19R, G142D, del156-157, R158G,

W258L, K417N, L452R, T478K, D614G, P681R, D950N), B.1.621 (T95I, insert144T, Y144S,

S373P, S375F, K417N, N440K, G446S, S477N, T478K, E484A, Q493R, G496S, Q498R, N501Y,

415 Pangolin GD_GenBank: QIA48632.1; Pangolin_GX-P2V_QIQ54048.1), SARS-CoV and related 
SARS CoV 007/2004 S_GenBank: AAU04646.1; WIV1_GenBank: KF367457;

SHC014_GenBank: KC881005), MERS-CoV EMC_GenBank: AFS88936), hCoV-HKU1

(GenBank: AAT98580.1), hCoV-OC43 (GenBank: AAT84354.1), hCoV-NL63 (GenBank:

Q6Q1S2.1) and hCoV-229E (GenBank: AOG74783.1) were synthesized (Genscript). These fulllength spike plasmids were used for pseudovirus production and for cell surface binding assays.

\section{Analysis of WS6 binding to cell surface expressed spike protein}

Expi-293 cells were transiently transfected with plasmids encoding full-length spike proteins of coronaviruses using Turbo293 transfection reagent (Speed BioSystems) following manufacturer's protocol. After 40 hours, cells were harvested and incubated with monoclonal antibodies $(1 \mu \mathrm{g} / \mathrm{ml})$ for 30 minutes. Cells were washed and incubated with an allophycocyanin conjugated anti-human IgG (709-136-149, Jackson Immunoresearch Laboratories) for another 30 minutes, then washed and fixed with $1 \%$ paraformaldehyde (15712-S, Electron Microscopy Sciences). Flow cytometry data were acquired in a BD LSRFortessa X-50 flow cytometer (BD biosciences) and analyzed using Flowjo (BD biosciences).

\section{Pseudovirus neutralization assay}

434 Spike-containing lentiviral pseudovirions were produced by co-transfection of packaging plasmid pCMVdR8.2, transducing plasmid pHR' CMV-Luc, a TMPRSS2 plasmid and S plasmids from human and animal coronaviruses (SARS-CoV-2 variants, SARS-CoV, MERS-CoV and SARSCoV-2, SARS-CoV related coronaviruses) into 293T cells using Lipofectamine 3000 transfection reagent (L3000-001, ThermoFisher Scientific, Asheville, NC) (Naldini et al., 1996). 293T-ACE2

439 cells (provided by Dr. Michael Farzan) or 293 flpin-TMPRSS2-ACE2 cells (made at the VRC) 
were plated into 96-well white/black Isoplates (PerkinElmer, Waltham, MA) at 75,00 cells per well the day before infection of pseudovirus. Serial dilutions of mAbs were mixed with titrated pseudovirus, incubated for 45 minutes at $37^{\circ} \mathrm{C}$ and added to cells in triplicate. Following $2 \mathrm{~h}$ of incubation, wells were replenished with $150 \mathrm{ml}$ of fresh media. Cells were lysed $72 \mathrm{~h}$ later, and luciferase activity was measured with Microbeta (Perking Elmer). Percent neutralization and neutralization IC50s, IC80s were calculated using GraphPad Prism 8.0.2.

\section{Neutralization by fusion inhibition (post attachment inhibition)}

BHK21-ACE2 or 293 flpin-TMPRSS2-ACE2 cells were placed on ice for one hour before to attach to ACE2. After 3 times of wash the cells were then incubated with mAbs on ice for additional one hour before returned to the incubator. Cells were lysed $72 \mathrm{~h}$ later, and luciferase activity was measured with Microbeta (Perking Elmer). Percent neutralization and neutralization

IC50s, IC80s were calculated using GraphPad Prism 8.0.2.

\section{Biolayer interferometry analysis of antibody binding affinity} spike proteins (S2Ps) were measured by biolayer interferometry using an Octet HTX instrument (Sartorius). All assays were performed in tilted 384-well plates (Geiger Bio-one) in HBS-EP+ buffer with agitation set to $1000 \mathrm{rpm}$ at $30^{\circ} \mathrm{C}$. The final volume for all solutions was $60-80 \mu 1 /$ well. Prior to running the assays, Anti Mouse Fc (AMC) biosensor tips were equilibrated in PBS for $\sim 5$ minutes. WS6 antibody IgG at $10 \mu \mathrm{g} / \mathrm{ml}$ were loaded onto AMC biosensors for 60s. Biosensors were then equilibrated in $\mathrm{HBS}-\mathrm{EP}+$ buffer for 60 s prior to measuring association with diverse beta- 
CoV S2P trimers in solution $(0.0019 \mu \mathrm{M}$ to $0.5 \mu \mathrm{M})$ for $300 \mathrm{~s}$; trimer proteins were then allowed to dissociate for 600s. Parallel correction to subtract systematic baseline drift was carried out by subtracting the measurements recorded for a sensor loaded with antibody incubated in HBS-EP+ buffer. Data analysis and curve fitting were carried out using Octet analysis software, version 12.0 (Sartorius). Experimental data were fitted using a 1:1 interaction to determine apparent dissociation constants and $\mathrm{k}_{\mathrm{on}}$ values.

\section{Crystallization and structure determination}

The N-terminal acetylated, C-terminal biotin-pegylated, 10-residue SARS-CoV-2 S2 peptide FKEELDKYFK (GenScript) was dissolved in 100\% DMSO at $10 \mathrm{mg} / \mathrm{mL}$ and then diluted with PBS to $1 \mathrm{mg} / \mathrm{mL}$. Fab WS6 was incubated with the peptide in a 1:2 molar ratio for 5 minutes to form complex. The Fab-peptide complex was concentrated to $10-15 \mathrm{mg} / \mathrm{mL}$ and screened for crystallization using 576 conditions from Hampton screen, Precipitant Synergy screen, and QIAGEN Wizard screen with a Mosquito robot mixing $100 \mathrm{~nL}$ reservoir solution and $100 \mathrm{~nL}$ protein solution per drop. Crystals were obtained from the solution containing 2\% v/v PEG 400, 2 $\mathrm{M}\left(\mathrm{NH}_{4}\right)_{2} \mathrm{SO}_{4}, 100 \mathrm{mM}$ sodium acetate $\mathrm{pH}$ 5.5. Crystal drops were set up in 15-well plates using $0.5 \mu \mathrm{L}$ WS6-peptide complex and $0.5 \mu \mathrm{L}$ of $80-100 \%$ concentration of the aforementioned nitrogen. ID22 and BM22). The diffraction data were indexed, integrated, and scaled with the HKL2000 crystallization solution. Crystals were cryoprotected in the aforementioned solution supplemented with $50 \%$ additional precipitant and $15 \% \mathrm{v} / \mathrm{v}(2 \mathrm{R}, 3 \mathrm{R})-2,3-$ Butanediol and flash frozen in liquid package (Otwinowski and Minor, 1997). The structures were determined by molecular 
486 replacement with Phaser (McCoy et al., 2007) in the CCP4 Program Suite (Collaborative

487 Computational Project, 1994). Further refinement was carried out with PHENIX (Adams et al.,

488 2010), starting with torsion-angle simulated annealing with slow cooling. Iterative manual model

489 building was carried out with COOT (Emsley and Cowtan, 2004) with maps generated from

490 combinations of standard positional, individual B-factor and TLS refinement algorithms. X-ray

491 data and refinement statistics are summarized in Table S2.

\section{Antibody germline assignment}

494 Germline gene of heavy and light chain were assigned by querying nucleotide sequences on

IGBLAST server. B6 heavy and light chain germline gene were assigned by submitting protein sequence. The $\mathrm{V}$ gene identity was reported as the identity to the germline nucleotide sequences.

\section{Antibody frequency calculation}

The mouse Ig deep sequencing samples were prepared and used to predict probability of downloaded from Bioproject PRJNA511481. The Ig sequences were sieved for IGOR to inference of having specific CDR3 sequences (Sethna et al., 2019). Signature of CV3-25 class antibody was defined as heavy chain IGHV5-51 and light chain IGKV1-12 germline gene, the R94, 96PQYC, G100b, C100d, and W100g on 18 amino acids CDR H3. The frequency of S2P6 class antibody was calculated by defining signature as IGHV1-46 germline gene with 97PKG in 11 amino acids

507 CDR H3, and IGKV3-20 with Y91, 93SSPP and 96F on 11 amino acids CDR L3. The frequency 508 of cc40.8 class antibody was calculated by masking IGHV3-23 with 94ITMA, 101V and 102V on 
8 amino acids CDR H3 and IGLV3-10 with T91, 94SGN, and A96 on 11 amino acids CDR L3.

510 The frequency of WS6 class antibody calculated by using mouse IGHV1-5 gene with 95TGS on 7

511 amino acids CDR H3, and IGKV4-61 gene with 91Y, 94Y and 96P on 9 amino acids CDR L3. B6

512 and IgG22 used identical heavy chain germline gene, although the light chain used different

513 germline genes, it shared similar contact residues. B6/IgG22 class frequency was calculated by

514 IGHV1-19 germline gene with $\mathrm{CX}\{5,10\} \mathrm{RX}\{4,6\} \mathrm{W}$ signature on CDR H3 amino acids, and

IGKV8-27 /IGKV1-99 germline genes with 92[LN] and 95[FY] on 8 amino acids length CDR L3.

\section{Phylogenetic tree}

Alpha-coronavirus spike sequences were downloaded using accession codes (NL63:Q6Q1S2.1, 229E:AOG74783.1, Feline:AAY32596.1, Mink:ADI80513.1). Beta-coronavirus spike sequences were downloaded from NCBI server using accession codes listed in Full-length spike constructs. ClustalW was used to calculate neighbor joining (NJ) tree (Larkin et al., 2007), and Dendroscope was used to plot the Neighbor Joining tree (Albrecht et al., 2012).

\section{QUANTIFICATION AND STATISTICAL ANALYSIS}

The BLI data were analyzed and plotted using GraphPad Prism. Crystal diffraction data and structural refinement statistics were analyzed with HKL2000, Phenix, and Molprobity. 


\section{References}

Adams, P.D., Afonine, P.V., Bunkoczi, G., Chen, V.B., Davis, I.W., Echols, N., Headd, J.J., Hung, L.W., Kapral, G.J., Grosse-Kunstleve, R.W., et al. (2010). PHENIX: a comprehensive Pythonbased system for macromolecular structure solution. Acta Crystallogr D Biol Crystallogr 66, 21321.

Albrecht, B., Scornavacca, C., Cenci, A., and Huson, D.H. (2012). Fast computation of minimum hybridization networks. Bioinformatics 28, 191-7.

Araf, Y., Akter, F., Tang, Y.D., Fatemi, R., Parvez, S.A., Zheng, C., and Hossain, G. (2022). Omicron variant of SARS-CoV-2: Genomics, transmissibility, and responses to current COVID-19 vaccines. J Med Virol, 10.1002/jmv.27588.

Barnes, C.O., West, A.P., Jr., Huey-Tubman, K.E., Hoffmann, M.A.G., Sharaf, N.G., Hoffman, P.R., Koranda, N., Gristick, H.B., Gaebler, C., Muecksch, F., et al. (2020). Structures of Human Antibodies Bound to SARS-CoV-2 Spike Reveal Common Epitopes and Recurrent Features of Antibodies. Cell 182, 828-842 e16.

Barouch, D.H., Yang, Z.Y., Kong, W.P., Korioth-Schmitz, B., Sumida, S.M., Truitt, D.M., Kishko, M.G., Arthur, J.C., Miura, A., Mascola, J.R., et al. (2005). A human T-cell leukemia virus type 1 regulatory element enhances the immunogenicity of human immunodeficiency virus type 1 DNA vaccines in mice and nonhuman primates. J Virol 79, 8828-34.

Boyoglu-Barnum, S., Ellis, D., Gillespie, R.A., Hutchinson, G.B., Park, Y.J., Moin, S.M., Acton, O.J., Ravichandran, R., Murphy, M., Pettie, D., et al. (2021). Quadrivalent influenza nanoparticle vaccines induce broad protection. Nature 592, 623-628.

Brouwer, P.J.M., Caniels, T.G., van der Straten, K., Snitselaar, J.L., Aldon, Y., Bangaru, S., Torres, J.L., Okba, N.M.A., Claireaux, M., Kerster, G., et al. (2020). Potent neutralizing antibodies from COVID-19 patients define multiple targets of vulnerability. Science 369, 643-650.

Cai, Y., Zhang, J., Xiao, T., Peng, H., Sterling, S.M., Walsh, R.M., Rawson, S., Rits-Volloch, S., and Chen, B. (2020). Distinct conformational states of SARS-CoV-2 spike protein. Science, eabd4251.

Cao, Y., Su, B., Guo, X., Sun, W., Deng, Y., Bao, L., Zhu, Q., Zhang, X., Zheng, Y., Geng, C., et al. (2020). Potent neutralizing antibodies against SARS-CoV-2 identified by high-throughput single-cell sequencing of convalescent patients' B cells. Cell, 10.1016/j.cell.2020.05.025.

Cerutti, G., Guo, Y., Zhou, T., Gorman, J., Lee, M., Rapp, M., Reddem, E.R., Yu, J., Bahna, F., Bimela, J., et al. (2021). Potent SARS-CoV-2 neutralizing antibodies directed against spike Nterminal domain target a single supersite. Cell Host Microbe 29, 819-833 e7.

563 Collaborative Computational Project, N. (1994). The CCP4 suite: programs for protein crystallography. Acta Crystallogr D Biol Crystallogr 50, 760-3. 
Corbett, K.S., Edwards, D.K., Leist, S.R., Abiona, O.M., Boyoglu-Barnum, S., Gillespie, R.A., Himansu, S., Schafer, A., Ziwawo, C.T., DiPiazza, A.T., et al. (2020). SARS-CoV-2 mRNA vaccine design enabled by prototype pathogen preparedness. Nature 586, 567-571.

Doria-Rose, N.A., Shen, X., Schmidt, S.D., O'Dell, S., McDanal, C., Feng, W., Tong, J., Eaton, A., Maglinao, M., Tang, H., et al. (2021). Booster of mRNA-1273 Strengthens SARS-CoV-2 Omicron Neutralization. medRxiv, 10.1101/2021.12.15.21267805.

Drosten, C., Gunther, S., Preiser, W., van der Werf, S., Brodt, H.R., Becker, S., Rabenau, H., Panning, M., Kolesnikova, L., Fouchier, R.A., et al. (2003). Identification of a novel coronavirus in patients with severe acute respiratory syndrome. N Engl J Med 348, 1967-76.

Edara, V.V., Manning, K.E., Ellis, M., Lai, L., Moore, K.M., Foster, S.L., Floyd, K., DavisGardner, M.E., Mantus, G., Nyhoff, L.E., et al. (2021). mRNA-1273 and BNT162b2 mRNA vaccines have reduced neutralizing activity against the SARS-CoV-2 Omicron variant. bioRxiv, 10.1101/2021.12.20.473557.

Emsley, P., and Cowtan, K. (2004). Coot: model-building tools for molecular graphics. Acta Crystallogr D Biol Crystallogr 60, 2126-32.

Garcia-Beltran, W.F., St Denis, K.J., Hoelzemer, A., Lam, E.C., Nitido, A.D., Sheehan, M.L., Berrios, C., Ofoman, O., Chang, C.C., Hauser, B.M., et al. (2022). mRNA-based COVID-19 vaccine boosters induce neutralizing immunity against SARS-CoV-2 Omicron variant. Cell, 10.1016/j.cell.2021.12.033.

Hsieh, C.L., Werner, A.P., Leist, S.R., Stevens, L.J., Falconer, E., Goldsmith, J.A., Chou, C.W., Abiona, O.M., West, A., Westendorf, K., et al. (2021). Stabilized coronavirus spike stem elicits a broadly protective antibody. Cell Rep 37, 109929.

Ju, B., Zhang, Q., Ge, J., Wang, R., Sun, J., Ge, X., Yu, J., Shan, S., Zhou, B., Song, S., et al. (2020). Human neutralizing antibodies elicited by SARS-CoV-2 infection. Nature 584, 115-119.

Kanekiyo, M., Wei, C.J., Yassine, H.M., McTamney, P.M., Boyington, J.C., Whittle, J.R., Rao, S.S., Kong, W.P., Wang, L., and Nabel, G.J. (2013). Self-assembling influenza nanoparticle vaccines elicit broadly neutralizing H1N1 antibodies. Nature 499, 102-6.

Ke, Z., Oton, J., Qu, K., Cortese, M., Zila, V., McKeane, L., Nakane, T., Zivanov, J., Neufeldt, C.J., Cerikan, B., et al. (2020). Structures and distributions of SARS-CoV-2 spike proteins on intact virions. Nature 588, 498-502.

Kong, R., Duan, H., Sheng, Z., Xu, K., Acharya, P., Chen, X., Cheng, C., Dingens, A.S., Gorman, J., Sastry, M., et al. (2019). Antibody Lineages with Vaccine-Induced Antigen-Binding Hotspots Develop Broad HIV Neutralization. Cell 178, 567-584 e19.

Kong, R., Xu, K., Zhou, T., Acharya, P., Lemmin, T., Liu, K., Ozorowski, G., Soto, C., Taft, J.D., Bailer, R.T., et al. (2016). Fusion peptide of HIV-1 as a site of vulnerability to neutralizing antibody. Science 352, 828-33. 
601

Ksiazek, T.G., Erdman, D., Goldsmith, C.S., Zaki, S.R., Peret, T., Emery, S., Tong, S., Urbani, C., Comer, J.A., Lim, W., et al. (2003). A novel coronavirus associated with severe acute respiratory syndrome. N Engl J Med 348, 1953-66.

Larkin, M.A., Blackshields, G., Brown, N.P., Chenna, R., McGettigan, P.A., McWilliam, H., Valentin, F., Wallace, I.M., Wilm, A., Lopez, R., et al. (2007). Clustal W and Clustal X version 2.0. Bioinformatics 23, 2947-8.

Li, W., Chen, Y., Prevost, J., Ullah, I., Lu, M., Gong, S.Y., Tauzin, A., Gasser, R., Vezina, D., Anand, S.P., et al. (2022). Structural basis and mode of action for two broadly neutralizing antibodies against SARS-CoV-2 emerging variants of concern. Cell Rep 38, 110210.

Liu, L., Iketani, S., Guo, Y., Chan, J.F., Wang, M., Liu, L., Luo, Y., Chu, H., Huang, Y., Nair, M.S., et al. (2021). Striking Antibody Evasion Manifested by the Omicron Variant of SARS-CoV2. Nature, 2021.12.14.472719.

Liu, L., Wang, P., Nair, M.S., Yu, J., Rapp, M., Wang, Q., Luo, Y., Chan, J.F., Sahi, V., Figueroa, A., et al. (2020). Potent neutralizing antibodies against multiple epitopes on SARS-CoV-2 spike. Nature 584, 450-456.

Marcou, Q., Mora, T., and Walczak, A.M. (2018). High-throughput immune repertoire analysis with IGoR. Nat Commun 9, 561.

McCallum, M., De Marco, A., Lempp, F.A., Tortorici, M.A., Pinto, D., Walls, A.C., Beltramello, M., Chen, A., Liu, Z., Zatta, F., et al. (2021). N-terminal domain antigenic mapping reveals a site of vulnerability for SARS-CoV-2. Cell 184, 2332-2347 e16.

McCoy, A.J., Grosse-Kunstleve, R.W., Adams, P.D., Winn, M.D., Storoni, L.C., and Read, R.J. (2007). Phaser crystallographic software. J Appl Crystallogr 40, 658-674.

Naldini, L., Blomer, U., Gage, F.H., Trono, D., and Verma, I.M. (1996). Efficient transfer, integration, and sustained long-term expression of the transgene in adult rat brains injected with a lentiviral vector. Proc Natl Acad Sci U S A 93, 11382-8.

Otwinowski, Z., and Minor, W. (1997). Processing of X-ray diffraction data collected in oscillation mode. Methods Enzymol 276, 307-26.

Pinto, D., Sauer, M.M., Czudnochowski, N., Low, J.S., Tortorici, M.A., Housley, M.P., Noack, J., Walls, A.C., Bowen, J.E., Guarino, B., et al. (2021). Broad betacoronavirus neutralization by a stem helix-specific human antibody. Science 373, 1109-1116.

Robbiani, D.F., Gaebler, C., Muecksch, F., Lorenzi, J.C.C., Wang, Z., Cho, A., Agudelo, M., Barnes, C.O., Gazumyan, A., Finkin, S., et al. (2020). Convergent antibody responses to SARSCoV-2 in convalescent individuals. Nature 584, 437-442.

Rogers, T.F., Zhao, F., Huang, D., Beutler, N., Burns, A., He, W.T., Limbo, O., Smith, C., Song, G., Woehl, J., et al. (2020). Isolation of potent SARS-CoV-2 neutralizing antibodies and protection from disease in a small animal model. Science 369, 956-963. 
Sauer, M.M., Tortorici, M.A., Park, Y.J., Walls, A.C., Homad, L., Acton, O.J., Bowen, J.E., Wang, C., Xiong, X., de van der Schueren, W., et al. (2021). Structural basis for broad coronavirus neutralization. Nat Struct Mol Biol 28, 478-486.

Sethna, Z., Elhanati, Y., Callan, C.G., Walczak, A.M., and Mora, T. (2019). OLGA: fast computation of generation probabilities of $\mathrm{B}$ - and $\mathrm{T}$-cell receptor amino acid sequences and motifs. Bioinformatics 35, 2974-2981.

Seydoux, E., Homad, L.J., MacCamy, A.J., Parks, K.R., Hurlburt, N.K., Jennewein, M.F., Akins, N.R., Stuart, A.B., Wan, Y.H., Feng, J., et al. (2020). Analysis of a SARS-CoV-2-Infected Individual Reveals Development of Potent Neutralizing Antibodies with Limited Somatic Mutation. Immunity 53, 98-105 e5.

Sievers, B.L., Chakraborty, S., Xue, Y., Gelbart, T., Gonzalez, J.C., Cassidy, A.G., Golan, Y., Prahl, M., Gaw, S.L., Arunachalam, P.S., et al. (2022). Antibodies elicited by SARS-CoV-2 infection or mRNA vaccines have reduced neutralizing activity against Beta and Omicron pseudoviruses. Sci Transl Med, eabn7842.

Suryadevara, N., Shrihari, S., Gilchuk, P., VanBlargan, L.A., Binshtein, E., Zost, S.J., Nargi, R.S., Sutton, R.E., Winkler, E.S., Chen, E.C., et al. (2021). Neutralizing and protective human monoclonal antibodies recognizing the N-terminal domain of the SARS-CoV-2 spike protein. Cell $184,2316-2331$ e15.

Syed, A.M., Ciling, A., Khalid, M.M., Sreekumar, B., Chen, P.Y., Kumar, G.R., Silva, I., Milbes, B., Kojima, N., Hess, V., et al. (2022). Omicron mutations enhance infectivity and reduce antibody neutralization of SARS-CoV-2 virus-like particles. medRxiv, 10.1101/2021.12.20.21268048.

Wang, L., Zhou, T., Zhang, Y., Yang, E.S., Schramm, C.A., Shi, W., Pegu, A., Oloniniyi, O.K., Henry, A.R., Darko, S., et al. (2021). Ultrapotent antibodies against diverse and highly transmissible SARS-CoV-2 variants. Science 373, eabh1766.

Wrapp, D., Wang, N., Corbett, K.S., Goldsmith, J.A., Hsieh, C.L., Abiona, O., Graham, B.S., and McLellan, J.S. (2020). Cryo-EM structure of the 2019-nCoV spike in the prefusion conformation. Science 367, 1260-1263.

Wu, X., Zhou, T., Zhu, J., Zhang, B., Georgiev, I., Wang, C., Chen, X., Longo, N.S., Louder, M., McKee, K., et al. (2011). Focused evolution of HIV-1 neutralizing antibodies revealed by structures and deep sequencing. Science 333, 1593-602.

Xu, K., Acharya, P., Kong, R., Cheng, C., Chuang, G.Y., Liu, K., Louder, M.K., O'Dell, S., Rawi, R., Sastry, M., et al. (2018). Epitope-based vaccine design yields fusion peptide-directed antibodies that neutralize diverse strains of HIV-1. Nat Med 24, 857-867.

Yassine, H.M., Boyington, J.C., McTamney, P.M., Wei, C.J., Kanekiyo, M., Kong, W.P., Gallagher, J.R., Wang, L., Zhang, Y., Joyce, M.G., et al. (2015). Hemagglutinin-stem nanoparticles generate heterosubtypic influenza protection. Nat Med 21, 1065-70. 
673 Zaki, A.M., van Boheemen, S., Bestebroer, T.M., Osterhaus, A.D., and Fouchier, R.A. (2012).

674 Isolation of a novel coronavirus from a man with pneumonia in Saudi Arabia. N Engl J Med 367,

675 1814-20.

Zhou, P., Yang, X.L., Wang, X.G., Hu, B., Zhang, L., Zhang, W., Si, H.R., Zhu, Y., Li, B., Huang, C.L., et al. (2020a). A pneumonia outbreak associated with a new coronavirus of probable bat 678 origin. Nature 579, 270-273.

679 Zhou, P., Yuan, M., Song, G., Beutler, N., Shaabani, N., Huang, D., He, W.T., Zhu, X., Callaghan, 680 S., Yong, P., et al. (2021). A protective broadly cross-reactive human antibody defines a conserved 681 site of vulnerability on beta-coronavirus spikes. bioRxiv, 10.1101/2021.03.30.437769.

682 Zhou, T., Teng, I.T., Olia, A.S., Cerutti, G., Gorman, J., Nazzari, A., Shi, W., Tsybovsky, Y., Wang, L., Wang, S., et al. (2020b). Structure-Based Design with Tag-Based Purification and InProcess Biotinylation Enable Streamlined Development of SARS-CoV-2 Spike Molecular Probes.

685 Cell Rep 33, 108322.

686 Zhu, N., Zhang, D., Wang, W., Li, X., Yang, B., Song, J., Zhao, X., Huang, B., Shi, W., Lu, R., et al. (2020). A Novel Coronavirus from Patients with Pneumonia in China, 2019. N Engl J Med 382, 688 727-733.

689 Zost, S.J., Gilchuk, P., Chen, R.E., Case, J.B., Reidy, J.X., Trivette, A., Nargi, R.S., Sutton, R.E., Suryadevara, N., Chen, E.C., et al. (2020). Rapid isolation and profiling of a diverse panel of human monoclonal antibodies targeting the SARS-CoV-2 spike protein. Nat Med 26, 1422-1427. 


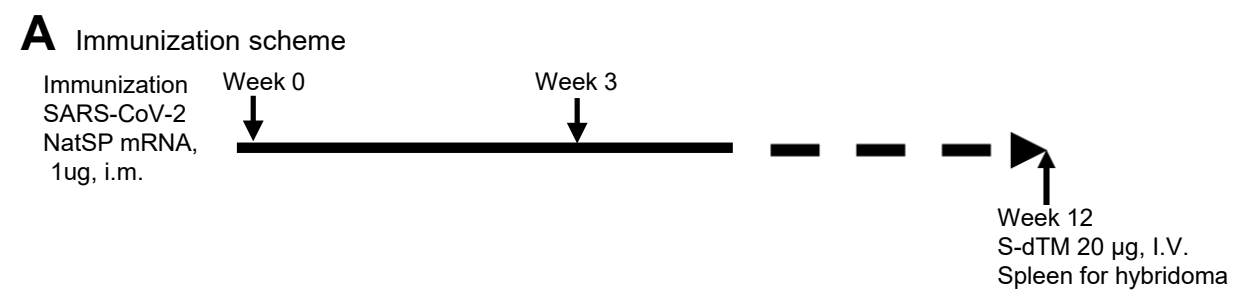

B SARS-CoV-2 spike-domain binding of isolated antibodies by ELISA

S-dTM
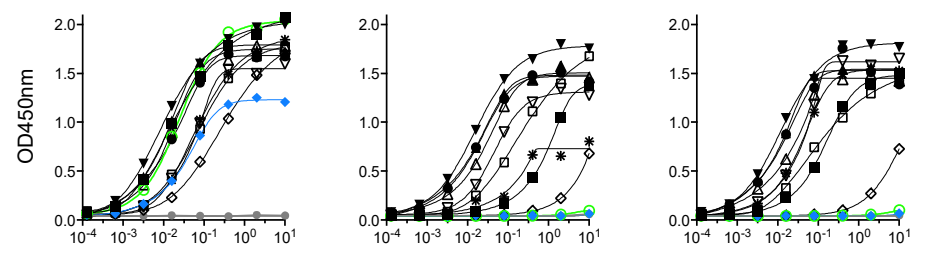

Antibody concentration $(\mu \mathrm{g} / \mathrm{ml})$
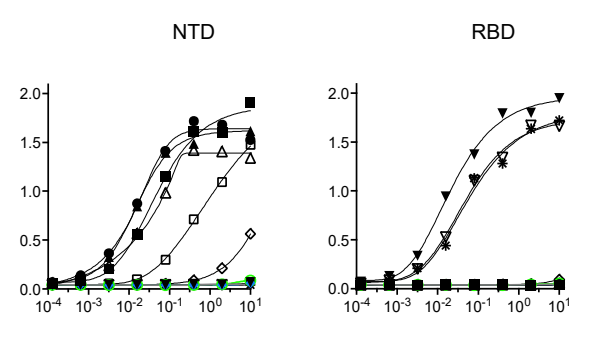

- WS1

WS2

W WS3

$\leftarrow$ WS5 - WS6 ๑ WS7 $\triangle$ WS8 $\nabla$ WS9

$\diamond$ WS10

*-WS11

$\rightarrow$ mouselgG1

C Divergent S2P binding of isolated antibodies by ELISA
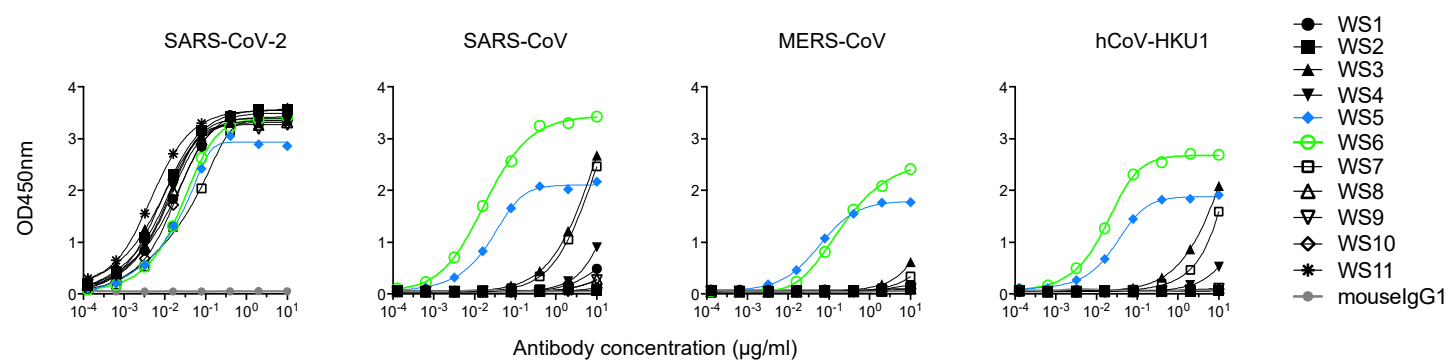

Antibody concentration $(\mu \mathrm{g} / \mathrm{ml})$

D SARS-CoV-2 neutralization assessments of WS5 and WS6

\begin{tabular}{|c|c|c|c|c|c|}
\hline \multirow[b]{2}{*}{ Abs } & \multicolumn{2}{|c|}{ SARS-CoV-2 WA-1 } & \multicolumn{2}{|c|}{ SARS-CoV } & \\
\hline & IC50 & IC80 & IC50 & IC80 & \\
\hline WS1 & $>50$ & $>50$ & $>50$ & $>50$ & \\
\hline wS2 & $>50$ & $>50$ & $>50$ & $>50$ & \\
\hline WS3 & 0.35 & 11.1 & $>50$ & $>50$ & \\
\hline WS4 & 0.08 & 0.10 & $>50$ & $>50$ & \\
\hline WS5 & $>50$ & $>50$ & $>50$ & $>50$ & \\
\hline WS6 & 0.16 & 4.07 & 0.76 & 12.5 & \\
\hline WS7 & 0.08 & 1.20 & $>50$ & $>50$ & $\mu \mathrm{g} / \mathrm{ml}$ \\
\hline WS8 & 0.12 & 10.0 & $>50$ & $>50$ & $0.1-1$ \\
\hline WS9 & 0.35 & $>50$ & $>50$ & $>50$ & $1-10$ \\
\hline WS10 & 0.61 & 17.2 & $>50$ & $>50$ & $10-50$ \\
\hline WS11 & $>50$ & $>50$ & $>50$ & $>50$ & $>50$ \\
\hline
\end{tabular}

Figure 1. Spike mRNA immunized mice elicit antibodies against diverse regions of spike, several of which bound diverse beta-coronavirus spikes and one of which, WS6, neutralized.

(A) Immunization scheme. NatSP is the full transmembrane-containing native sequence of spike WA-1 strain; S-dTM is the soluble spike protein residues 1-1206 of wildtype WA-1 strain. (B) Binding of isolated antibodies by ELISA to prefusionstabilized spike (S2P) and its subdomains. Purified monoclonal antibodies from hybridoma supernatants were analyzed for binding to SARS-CoV-2 S-dTM, S1 (S1-short and S1R), RBD and NTD by ELISA. (C) Binding of isolated antibodies assess by ELISA to diverse beta-coronavirus prefusion-stabilized spikes (S2P). (D) Neutralization assessment of hybridoma antibodies against SARSCoV-2 WA-1 and SARS-CoV pseudovirus on 293T-ACE2 cells. See also Figure S1. 
A WS6 binding to CoV spike proteins by ELISA

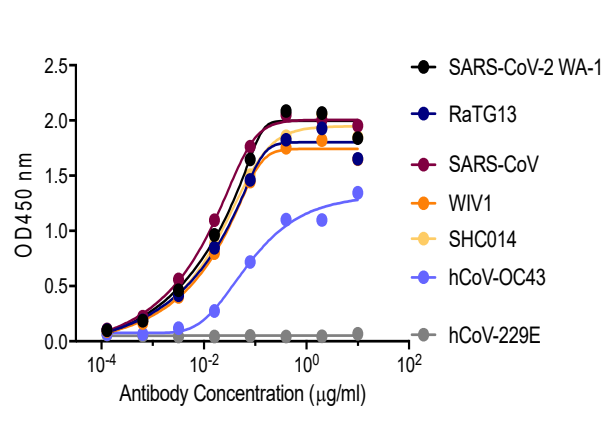

C WS6 binding to cell surface expressed spikes SARS-CoV-2_WA-1
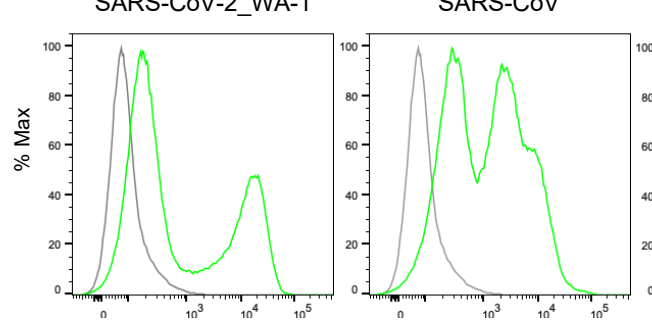

B WS6 IgG binding on-rates to diverse CoV spikes by Bio-Layer Interferometry
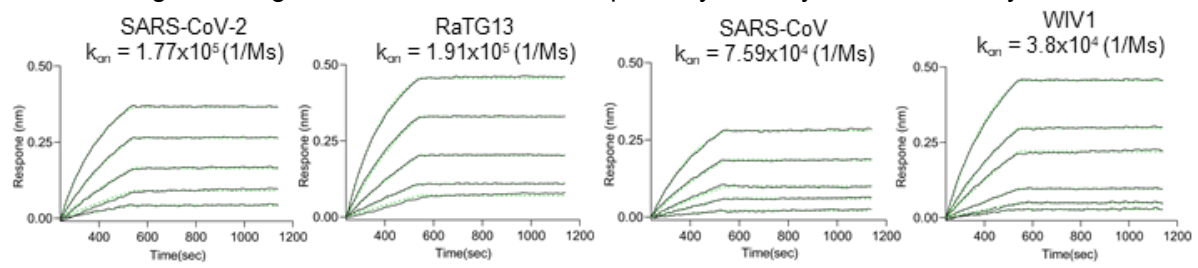

$$
\mathrm{SCH} 014
$$

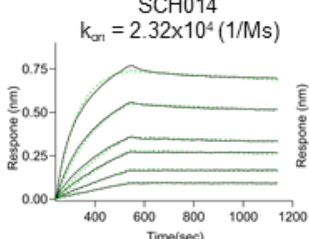

$\mathrm{hCoV}-\mathrm{OC} 43$
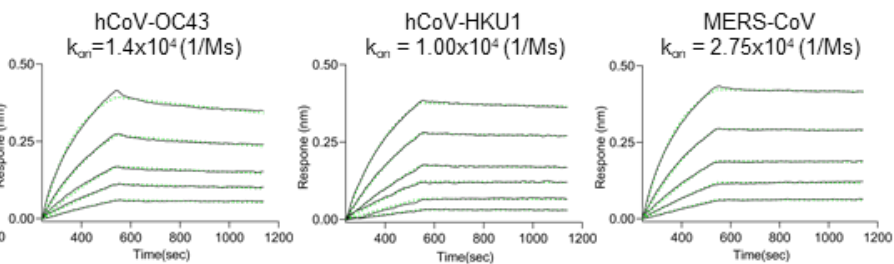

D WS6 neutralization of pseudoviruses SARS-CoV-2 variants
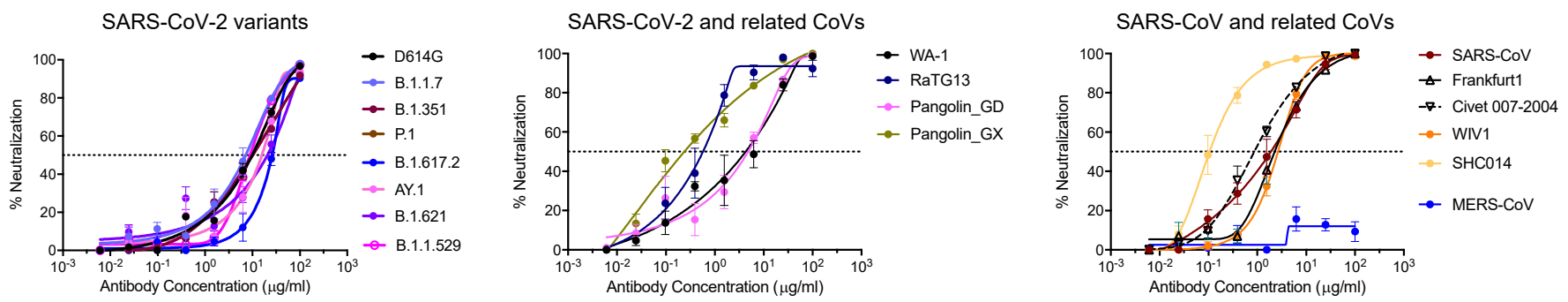

Figure 2. Antibody WS6 binds and neutralizes diverse beta-coronaviruses.

(A) ELISA binding analysis of WS6 to prefusion-stabilized soluble spikes of various coronaviruses. (B) BLI binding curves of WS6 with various beta-coronaviruses. (C) WS6 binds cell surface-expressed spike proteins from SARS-CoV-2, SARS-CoV, hCoV-HKU1, hCoV-OC43 and MERS-CoV. (D) WS6 neutralizes SARS-CoV-2 variants, SARS-CoV and related animal coronaviruses. Neutralization activity was measured using spike-pseudotyped lentivirus on 293 flpin-TMPRSS2-ACE2 cells. Assays were performed in triplicate on 293 flpin-TMPRSS2-ACE2 cells, and representative neutralization curves from 2-3 technical replicates are shown. (Left) WS6 neutralizes SARS-CoV-2 variants, D614G, B.1.1.7 (Alpha), B.1.351 (Beta), P.1 (Gamma), B.1.617.2 (Delta), AY.1 (Delta+), B.1.621 (Mu) and B.1.1.529 (Omicron). (Middle) WS6 neutralizes SARS-CoV-2 and related coronaviruses, SARS-CoV-2 WA-1, RaTG13, Pangolin_GD and Pangolin_GX. (Right) WS6 neutralizes SARS-CoV and related coronaviruses, SARS-CoV, Frankfurt1, Civet 007-2004, WIV1 and SHC014, but not MERS-CoV. See also Figure S1, S2, S3 and Table S1. 
A Negative stain EM of WS6 in complex with SARS-CoV-2 spike

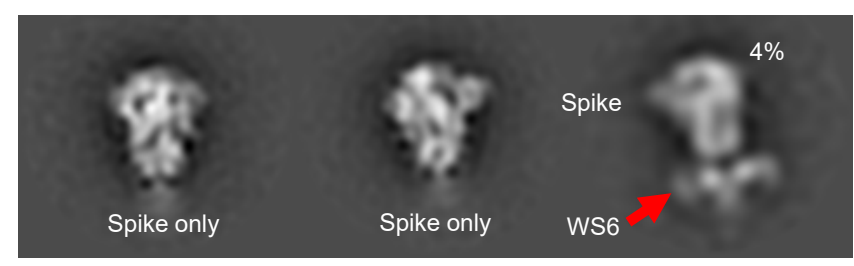

B Negative stain EM of WS6 in complex with SARS-CoV-2 spike S2

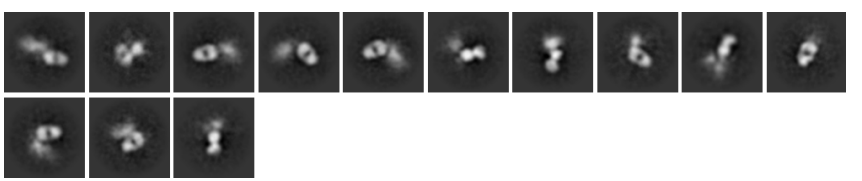

D Crystal structure of WS6 in complex with S2 peptide at $2.0 \AA$ resolution
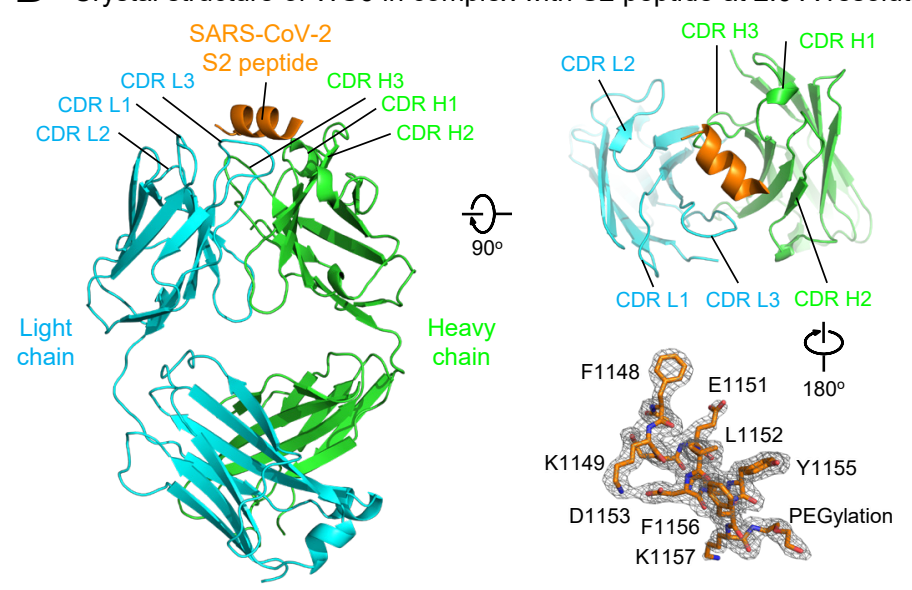

C Mapping the epitope with a SARS-CoV spike peptide array

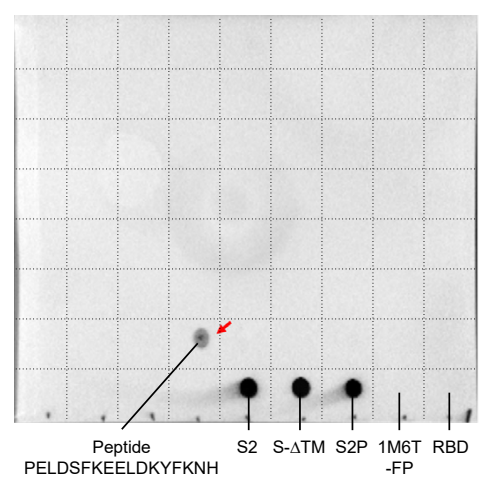

E Interactions between WS6 and S2 peptide

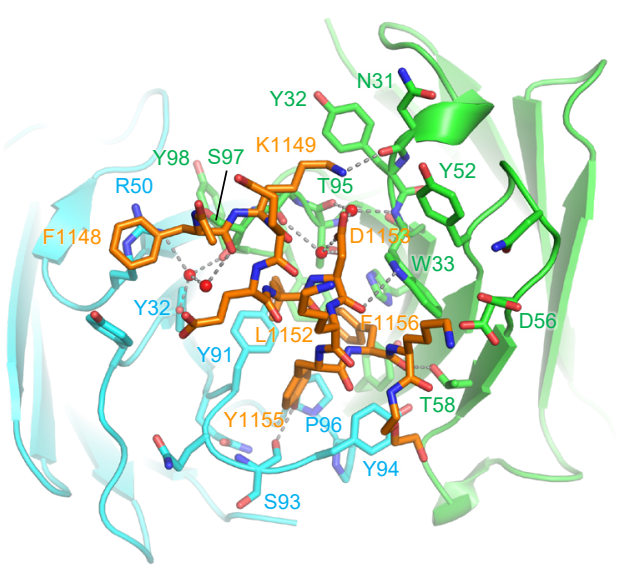

F ws6 sequence and paratope

Heavy chain

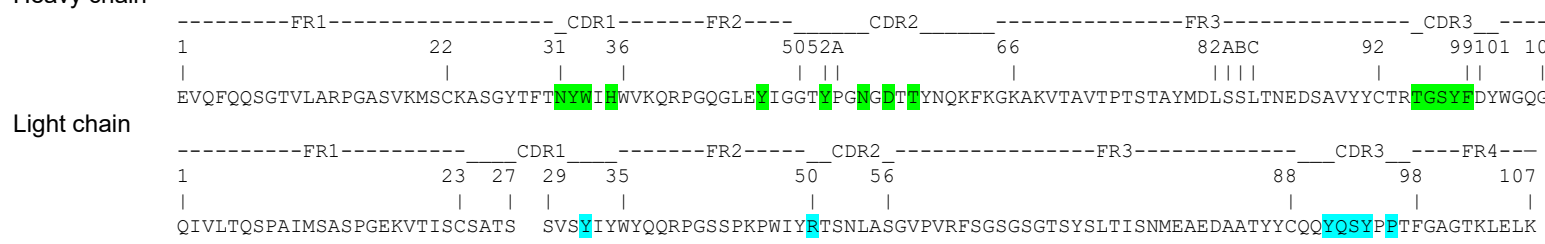

Figure 3. Epitope mapping and crystal structure of antibody WS6 in complex with S2 peptide.

(A) Negative stain EM of SARS-CoV-2 spike in complex with WS6. Only a small fraction was observed with WS6 bound at the stem region. (B) Negative stain EM of SARS-CoV-2 spike S2 in complex with WS6. (C) Epitope mapping by dot-blot assay with a SARS-CoV spike peptide array (BEI, NR-52418). WS6 bound to peptide \#154 $\mathrm{P}_{1125}$ ELDSFKEELDKYFKNH $_{1141}$

(corresponding to SARS-CoV-2 residues 1143-1159) (red arrow), SARS-CoV-2 spike and its S2 domain. A dash line grid was added on top of the blot to aid display. (D) Crystal structure of WS6 in complex with SARS-CoV-2 S2 peptide at $2.0 \AA$ resolution. The antibody and SARS-CoV-2 peptide are shown in cartoon representation. WS6 heavy chain, light chain and S2 peptide are colored green, cyan and orange, respectively. Two $90^{\circ}$-flipped views were shown (left, top right). Electron density map of the S2 peptide was shown with the antibody interacting residues facing the reader (right, bottom) in a $180^{\circ}$-flipped view from panel above. (E) Detailed interactions between WS6 and S2 peptide. The S2 peptide and paratope residues in WS6 were shown in sticks representation with other regions of WS6 shown in cartoon representation. Hydrogen bonds were indicated with gray dashed lines. Waters that mediating hydrogen bonds were shown as red spheres. (F) Sequence and paratope of WS6. WS6 residues were numbered according to Kabat nomenclature. Heavy and light chain paratope residues were highlighted in green and cyan, respectively. See also Table S3. 
A WS6 inhibits post viral attachment

BHK21-ACE2 cells

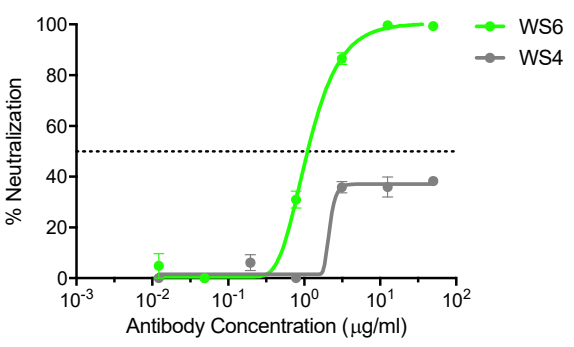

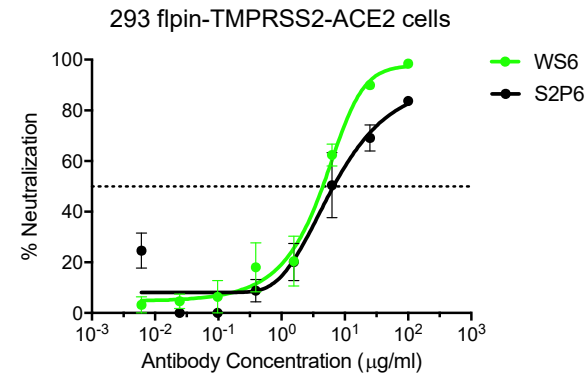

B WS6-binding is not compatible with the prefusion and postfusion conformation of the spike WS6 superposed onto prefusion conformation WS6 superposed onto the postfusion conformation

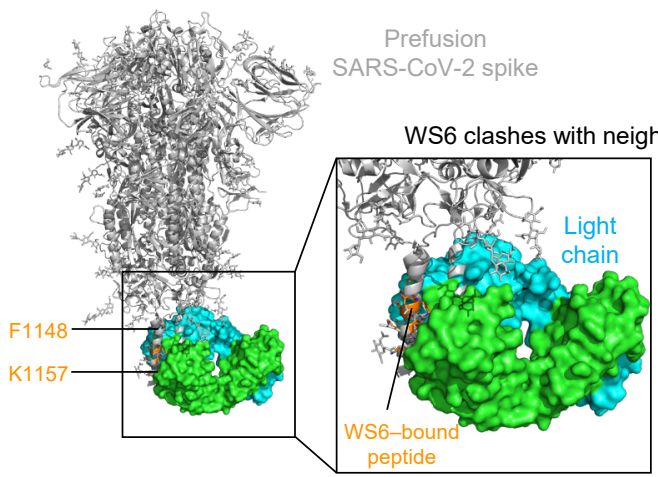

Postfusion Postfusion
RS-CoV-2 spike

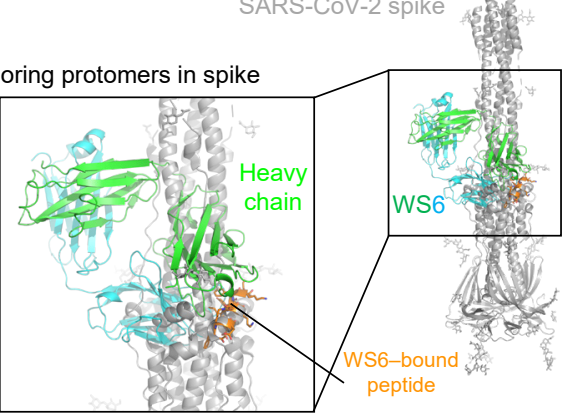

C Potential mechanism of neutralization

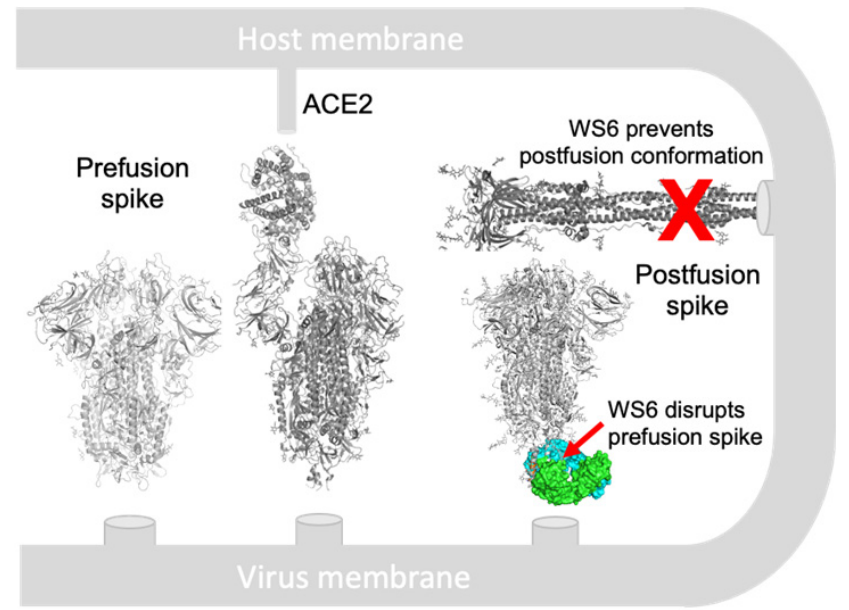

Figure 4. WS6 neutralizes SARS-CoV-2 by inhibition of post viral attachment.

(A) WS6 and S2P6 neutralization of SARS-CoV-2 by inhibition of post viral attachment. Neutralization experiments were performed with two BHK21-ACE2 (top) and 293 flpin-TMPRSS2-ACE2 (bottom) cells. WS4, a RBD antibody, was used as a negative control. Representative neutralization curves from two technical replicates of experiments are shown. (B) Binding mode of WS6 is not compatible with the prefusion and postfusion conformation of the spike. The WS6-S2 peptide complex was superposed onto the pre- and post-fusion spike trimer by aligning the WS6-bound S2 peptide with the corresponding part in one of the protomers. S2-bound WS6 clashed with neighboring protomers in either prefusion or postfusion conformation, suggesting binding disrupts prefusion conformation and prevents formation of postfusion. (C) Mechanism of neutralization by WS6. Structure of WS6 in complex with S2 peptide indicated that WS6 disrupts prefusion spike and prevents formation of the postfusion conformation. 
bioRxiv preprint doi: https://doi.org/10.1101/2022.01.25.477770; this version posted January 26, 2022. The copyright holder for this preprint (which was not certified by peer review) is the author/funder. This article is a US Government work. It is not subject to copyright under. 17 . USC 5
105 and is also made available for use under a CCO license.

A Comparison of binding orientations of S2-targeting antibodies

WS6 Ig 2 Ig622

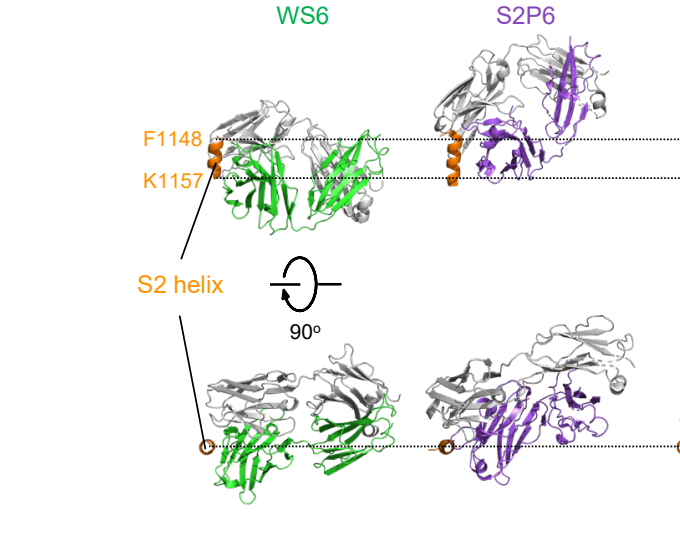

B6

$\mathrm{CC} 40.8$

CV3-25

B A supersite of vulnerability on the $\mathrm{S} 2$ stem
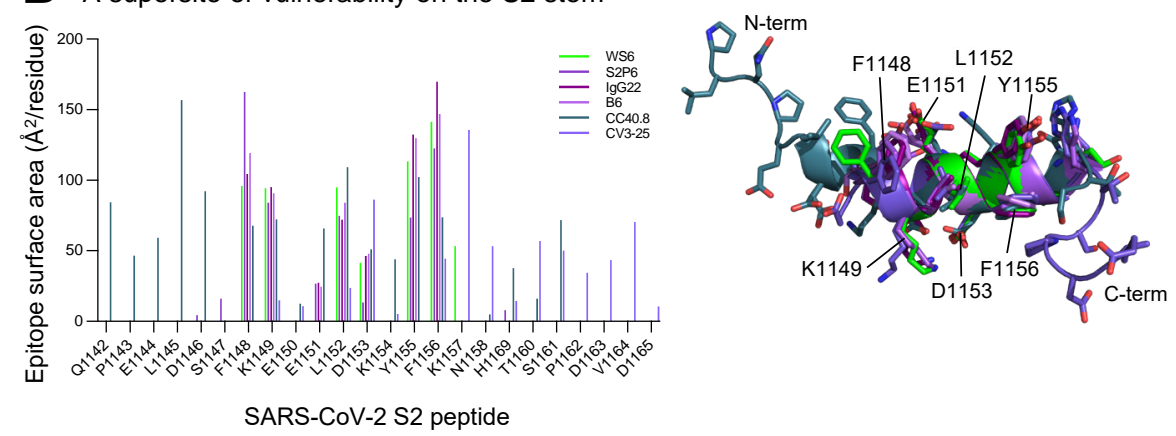

Supersite of vulnerability
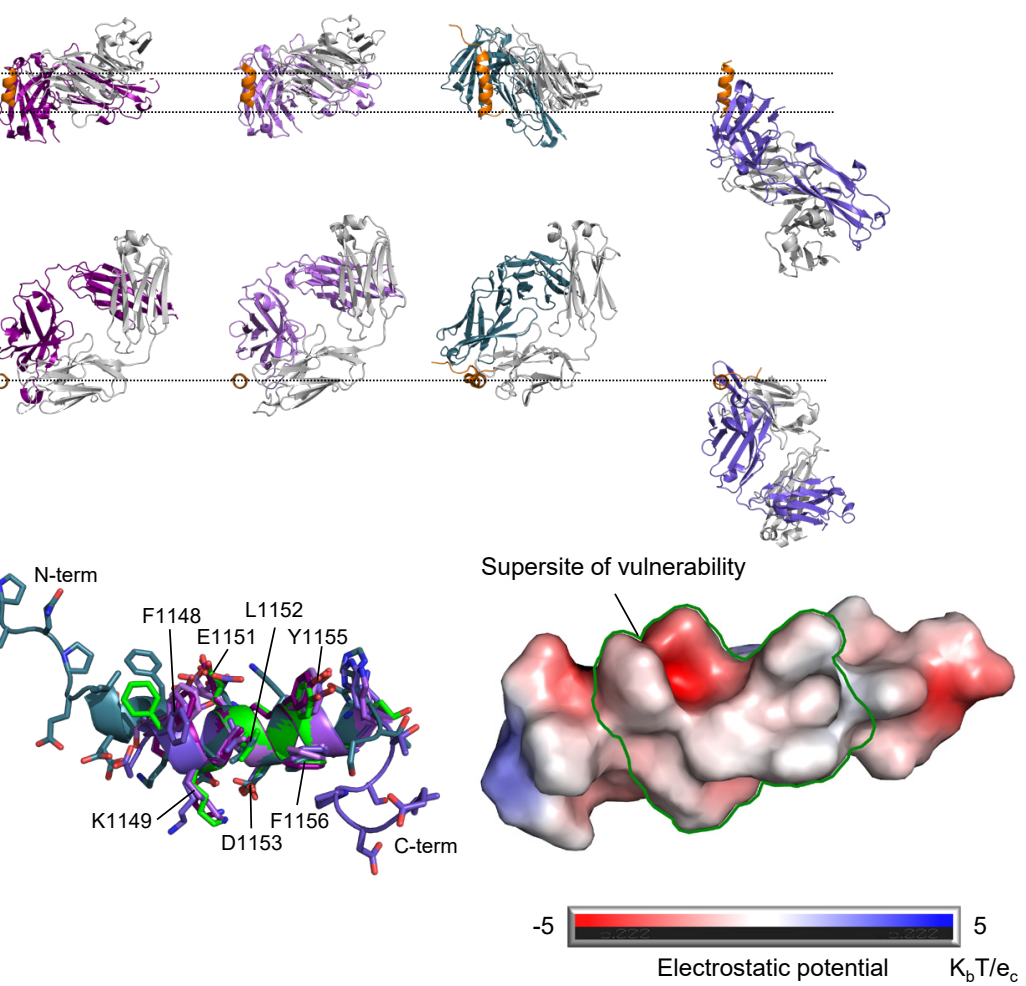

C Sequence conservation of the supersite in representative beta-coronaviruses
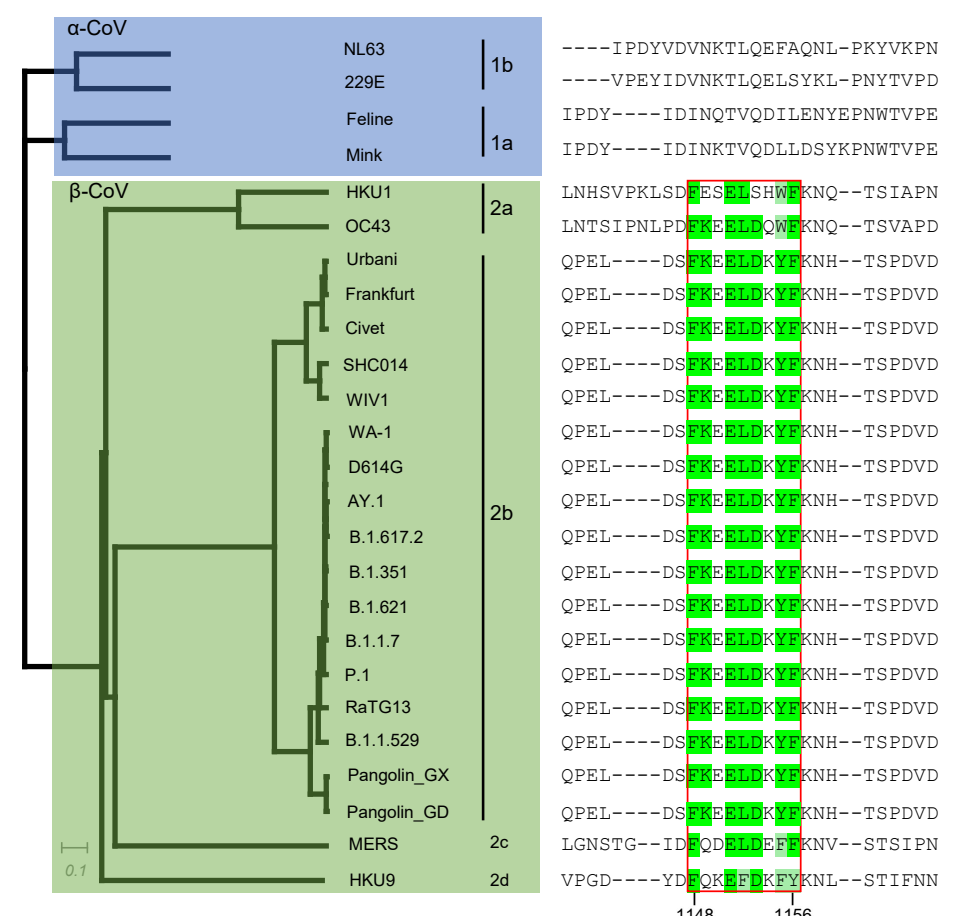

Figure 5. An S2-stem supersite of vulnerability.

(A) Comparison of modes of recognition of WS6 with other antibodies targeting the S2 stem helix. WS6 binding to the S2 helix was shown in the same orientation as in Fig. 4B. S2P6, IgG22, B6, CC40.8 and CV3-25 complexes were aligned with the WS6 complex over the S2 peptide helix. The S2 helix and light chains of antibodies were colored orange and gray, respectively. Heavy chains of antibodies are colored differently to distinguish. CV3-25 assumes a distinct mode of recognition comparing to others. (B) Epitopes of antibodies targeting the S2 region define a supersite of vulnerability. Antibody-binding surface areas of each residue in the S2 peptide were plotted along the linear sequence of SARS-CoV-2 S2 peptide (left). Antibody-bound peptides were shown colored by recognizing antibodies. The center region between residues 1148 and 1156 were recognized by most of the antibodies (middle), hydrophobic residues F1148, L1152, Y1155 and F1156 were positioned in the middle of the supersite with K1149, E1151 and D1153 providing hydrophilic interactions are the peripheral (right). The boundary of the supersite, which was defined as residues interacting with 5 of the 6 antibodies analyzed in this study, was highlighted by a green line. (C) Sequence conservation of the SARS-CoV-2 supersite in $\beta$-coronaviruses. Sequence dendrogram was calculated using full spike protein sequences (far left). Sequence alignment shown residues 1142 - 1165, recognized by supersite antibodies in panel B (left middle). The surface of S2 peptide was colored by sequence variation score with corresponding residues shown in sticks representation (right). See also Figures S4 and S5. 